\title{
Cancer targeted gene therapy for inhibition of melanoma lung metastasis with eIF3i shRNA loaded liposomes
}

Wen Xiao ${ }^{1}$, Weiyi Zhang ${ }^{1}$, Hai Huang ${ }^{1}$, Yafei Xie, Yi Zhang, Xia Guo, Chaohui Jin, Xuelian Liao, Shaohua Yao, Guo Chen *, Xiangrong Song *

State Key Laboratory of Biotherapy/Geriatrics and Cancer Center, West China Hospital, Sichuan University, and Collaborative Innovation Center for Biotherapy, Sichuan University, Chengdu, China

* Corresponding authors

Department of Anesthesiology, State Key Laboratory of Biotherapy and Cancer Center, West China Hospital, Sichuan University and Collaborative Innovation Center of Biotherapy, Chengdu 610041, China

E-mail: songxr@scu.edu.cn (Xiangrong Song); Grace_chenguo@hotmail.com (Guo Chen)

Tel./Fax: +86-28-8550-3817.

${ }^{1}$ These authors contributed equally to this article.

A
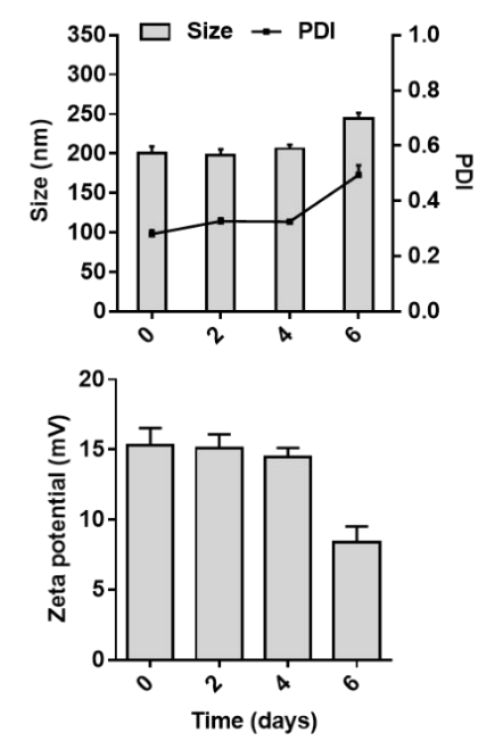

B
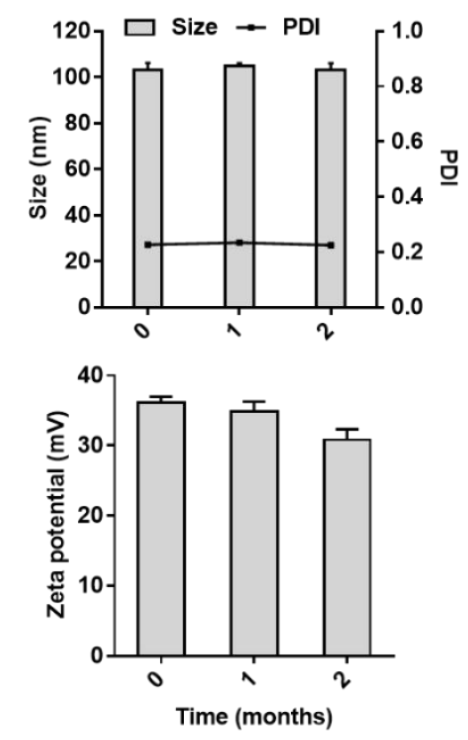

C
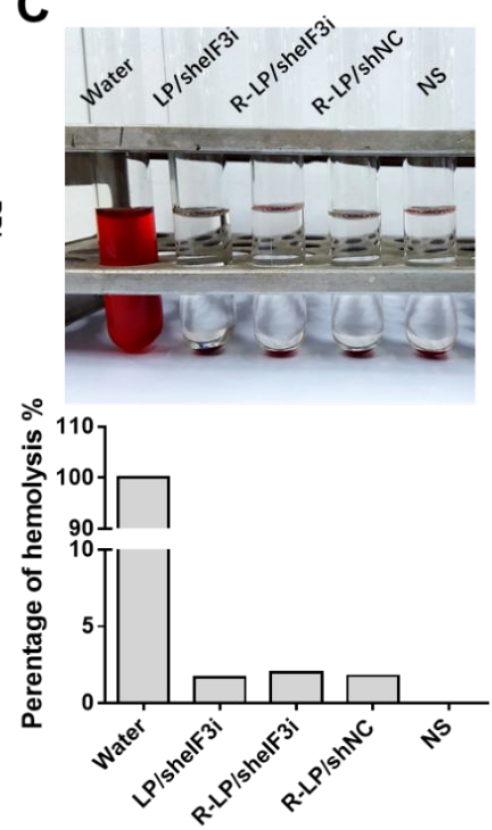

Supplementary Figure 1. Change of mean diameter, polydispersity index (PDI) and zeta potential of R-LP/sheIF3i (A) and R-LP (B) stored at $4{ }^{\circ} \mathrm{C}$. (C) Images of hemolysis on red blood cells and the percentage of hemolysis (\%) in each group. 


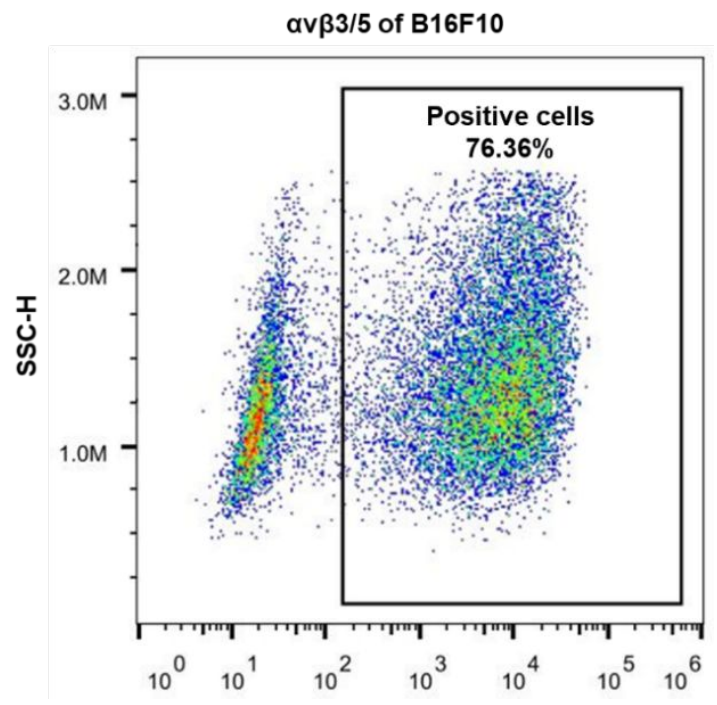

Supplementary Figure 2. Detection of integrin receptor expressed on melanoma cancer cells (B16F10 cells). $76.36 \%$ of B16F10 cells express $\alpha \mathrm{v} \beta 3 / 5$. 